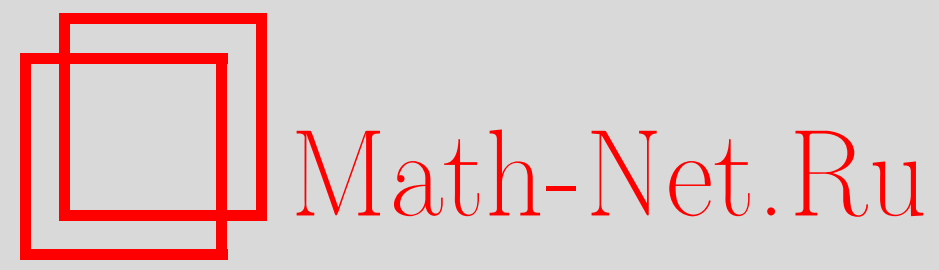

M. В. Бурнашев, Т. С. Хан, Шун-ичи Амари, О некоторых задачах оценивания с информационными ограничениями, Теория вероятн. и ее примен., 2001, том 46, выпуск 2, 233-246

DOI: https://doi.org/10.4213/tvp3916

Использование Общероссийского математического портала MathNet.Ru подразумевает, что вы прочитали и согласны с пользовательским соглашением

http://www . mathnet.ru/rus/agreement

Параметры загрузки:

IP : 35.173 .219 .12

26 апреля 2023 г., 09:56:46

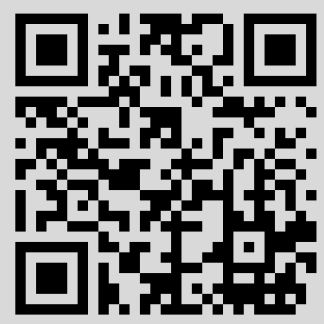


(c) $2001 \mathrm{r}$.

БУРНАШЕВ М. В., ХАН Т. С. $\stackrel{* *}{ }$ АМАРИ ШI.***

\section{О НЕКОТОРЫХ ЗАДАЧАХ ОЦЕНИВАНИЯ С ИНФОРМАЦИОННЫМИ ОГРАНИЧЕНИЯМИ ${ }^{1)}$}

Статья является второй частью работы авторов [1]. Рассматривается задача оценки параметра, в которой часть данных не может наблюдаться непосредственно. Имеющийся помощник наблюдает эти данные и может передать нам некоторую ограниченную информацию о них. Какая именно информация позволит достичь наименьшей среднеквадратичной ошибки при оценивании параметpa? В частности, какая минимальная информация достаточна для получения той же среднеквадратичной ошибки, как если бы мы непосредственно наблюдали все данные? Получены оценка сверху для величины этой минимальной информации и некоторые подобные результаты.

Ключевые слова и фразы: оценка параметра, среднеквадратичная ошибка, фишеровская информация, скорость передачи информации, критическая скорость.

Данная статья является второй частью работы авторов [1], из которой мы используем также обозначения.

Далее для модели ДСК («двоичный симметричный канал») из примера 2 [1] мы рассмотрим задачу оценивания параметра $p$.

Всюду далее $\log x=\log _{2} x$. Для любого множества $\mathscr{A}$ через $|\mathscr{A}|$ или $A$ обозначается его мошность. Для произвольной функции $f(x)$, $x \in \mathscr{A}$, через $|f|$ обозначается мощность множества $f(\mathscr{A})$.

1. Постановка задачи и некоторые вспомогательные результаты. Нам удобнее будет рассмотреть сначала более общую, чем для ДСК, задачу оценивания параметра. При этом, для разнообразия, в отличие от [1] мы поменяем местами статистика и помощника (т.е.

\footnotetext{
${ }^{*}$ Институт проблем передачи информашии РАН, Большой Каретный пер., 19, 101447 Москва, Россия; e-mail: burn@i.i.tp.ru

${ }^{* *}$ Graduate School of Information Systems, University of Electro-Communications, Chfugaoka 1-5-1, Chofu, Tokyo 182, Japan.

${ }^{* * *}$ RIKEN Brain Science Institute, Wako-shi, Hirosawa 2-1, Saitama 351-0198, Japan.

1) Работа выполнена при частичной финансовой поддержке Российского фонда фундаментальных исследований (номер проекта 98-01-04108).
} 
статистик будет наблюдать вход канала, а помощник наблюдает выход канала). Конечно, это не меняет сути задачи, однако делает более ясными ее связи с другими задачами.

Пусть $\mathscr{X}$ и $\mathscr{Y}$ суть конечные множества, представляющие входной и выходной алфавиты канала соответственно. Задана переходная вероятность $p(y \mid x, \theta), x \in \mathscr{X}, y \in \mathscr{Y}$, т.е. вероятность получить на выходе $y$ при условии, что на входе был $x$. Предполагается, что она зависит от неизвестного параметра $\theta \in[a, b] \subset \mathbf{R}^{1}$, подлежащего оцениванию. Задано также некоторое априорное распределение $\pi(x)$ на входном алфавите $\mathscr{X}^{n}$, и входной блок $x^{n}$ представляет собой вектор из $n$ независимых случайных величин с этим распределением $\pi(x)$. Будем обозначать его распределение через $\pi\left(x^{n}\right)$.

Пусть задана скорость передачи $R>0$ и разрешается заранее разбить выходное пространство $\mathscr{Y}^{n}$ на $N \leqslant 2^{R n}$ частей $\left\{\mathscr{Y}_{1}, \ldots, \mathscr{Y}_{N}\right\}$. Теперь статистик наблюдает только вход канала $x^{n}$, а помощник наблюдает только выход канала $y^{n}$. Далее помощник сообщает статистику, какой части $\mathscr{Y}_{i}$ принадлежит наблюдение $y^{n}$. Другими словами, на множестве $\mathscr{Y}^{n}$ выбирается произвольная функция $f\left(y^{n}\right)$, принимающая не более $N$ различных значений (т.е. $|\log f| \leqslant R n$ ). Основываясь на собственном наблюдении $x^{n}$ и дополнительной информации $f\left(y^{n}\right)$ от помощника, статистик строит некоторую оценку $\hat{\theta}_{n}=\hat{\theta}_{n}\left(x^{n}, f\left(y^{n}\right)\right)$ неизвестного параметра $\theta$. Нас будет интересовать асимптотика $(n \rightarrow \infty)$ среднеквадратичного риска оценки $\hat{\theta}_{n}$, а именно, поведение функции

$$
\begin{aligned}
e\left(\theta, \hat{\theta}_{n}, R, f\right) & =\limsup _{n \rightarrow \infty}\left\{n \mathbf{E}_{\theta}\left[\hat{\theta}_{n}\left(x^{n}, f\left(y^{n}\right)\right)-\theta\right]^{2}\right\} \\
& =\limsup _{n \rightarrow \infty}\left\{n \sum_{x^{n} \in \mathscr{X}^{n}} \pi\left(x^{n}\right) \mathbf{E}_{\theta}\left[\left(\hat{\theta}_{n}\left(x^{n}, f\left(y^{n}\right)\right)-\theta\right)^{2} \mid x^{n}\right]\right\},
\end{aligned}
$$

$R>0$, для хороших функций $f\left(y^{n}\right)$ (разбиений $\left.\left\{\mathscr{Y}_{i}\right\}\right)$ и хороших оценок $\hat{\theta}_{n}$.

Для этого естественно сначала понять поведение этой функции в случае, когда скорость передачи $R$ велика настолько, что помощник может сообщить статистику точное значение блока $y^{n}$ (например, $R \geqslant \log |\mathscr{Y}|)$. Тогда мы приходим к стандартной статистической задаче оценки параметра по наблюдениям $x^{n}, y^{n}$. Мы будем рассматривать регулярный случай этой задачи, когда переходная вероятность $p(y \mid x, \theta)$ является непрерывно дифференцируемой (по $\theta$ ) функцией для любых $x \in \mathscr{X}, y \in \mathscr{Y}$ и для нее определена фишеровская информация

$$
I(\theta)=\sum_{x \in \mathscr{X}} \pi(x) \sum_{y \in \mathscr{Y}} p^{2}(y \mid x, \theta) p(y \mid x, \theta)>0 .
$$

Так как $\mathscr{X}, \mathscr{Y}$ суть конечные множества, то функция $I(\theta)$ является равномерно непрерывной для $\theta \in[a, b]$. 
В стандартной теории оценивания всегда делается некоторое предположение, обеспечивающее существование какой-либо равномерно состоятельной оценки параметра $\theta[4],[5]$. Тогда задача сведется к исследованию свойств наилучших оценок $\hat{\theta}_{n}$ в малой окрестности истинного значения $\theta$. Для этого в нашем случае достаточно, например, предположить, что семейство вероятностных мер $\{p(y \mid x, \theta), \theta \in[a, b]\}$ является «равномерно разделенным» по $\theta$, т.е.

$$
\inf _{\left|\theta-\theta_{1}\right| \geqslant \varepsilon} \sum_{x \in \mathscr{X}} \pi(x) \sum_{y \in \mathscr{Y}}\left|p(y \mid x, \theta)-p\left(y \mid x, \theta_{1}\right)\right|>0 \quad \text { для любого } \varepsilon>0 .
$$

Пусть, далее, $\hat{\theta}_{n}\left(x^{n}, y^{n}\right)$ - оценка максимального правдоподобия параметра $\theta$. Из стандартной теории оценивания следует, что равномерно по $\theta \in[a, b]$ выполняется соотношение

$$
\lim _{n \rightarrow \infty}\left\{n \mathbf{E}_{\theta}\left[\hat{\theta}_{n}\left(x^{n}, f\left(y^{n}\right)\right)-\theta\right]^{2}\right\} \leqslant \frac{1}{I(\theta)}
$$

(неравенство в (4) возникает на концах отрезка $[a, b]$ ).

С другой стороны, известно, что для любых $\delta>0$ и $c \in[a, b]$ справедливо неравенство $(c \delta=(c-\delta, c+\delta) \cap[a, b])$

$$
\lim _{n \rightarrow \infty} \inf _{\tilde{\theta}_{n}} \sup _{\theta \in c_{\delta}}\left\{n \mathbf{E}_{\theta}\left[\tilde{\theta}_{n}\left(x^{n}, f\left(y^{n}\right)\right)-\theta\right]^{2}-\frac{1}{I(\theta)}\right\} \geqslant 0 .
$$

Поэтому из (4), (5) следует

$$
\lim _{n \rightarrow \infty} \inf _{\tilde{\theta}_{n}} \sup _{\theta \in[a, b]}\left\{n \mathbf{E}_{\theta}\left[\tilde{\theta}_{n}\left(x^{n}, y^{n}\right)-\theta\right]^{2}-\frac{1}{I(\theta)}\right\}=0 .
$$

Нас будет интересовать вопрос, при какой минимальной скорости $R_{\text {crit }}=R_{\text {crit }}([a, b])$ выполняется естественный аналог соотношения (6)? Другими словами, при каких скоростях $R$ существует функция $f\left(y^{n}\right)$ с $|\log f| \leqslant R n$ такая, что выполняется соотношение

$$
\lim _{n \rightarrow \infty} \inf _{\tilde{\theta}_{n}} \sup _{\theta \in[a, b]}\left\{n \mathbf{E}_{\theta}\left[\tilde{\theta}_{n}\left(x^{n}, f\left(y^{n}\right)\right)-\theta\right]^{2}-\frac{1}{I(\theta)}\right\}=0 .
$$

Покажем сначала, что достаточно установить соотношение (7) в некоторой локальной $\delta(n)$-окрестности каждой точки $\theta \in[a, b]$.

Предложение 1. Для справедливости соотношения (7) достаточно, чтобы для любого $c \in[a, b] u \delta(n)=(\ln n) / \sqrt{n}$ выполнялось соотношение

$$
\lim _{n \rightarrow \infty} \inf _{\tilde{\theta}_{n}} \sup _{\theta \in c_{\delta(n)}}\left\{n \mathbf{E}_{\theta}\left[\tilde{\theta}_{n}\left(x^{n}, f\left(y^{n}\right)\right)-\theta\right]^{2}-\frac{1}{I(\theta)}\right\}=0 .
$$


Д ок аз ат ельст в о. Представим себе, что статистик знает точную величину блока $y^{m}$ для некоторого $m<n$. Тогда в силу предположений $(2),(3)$ существуют положительные постоянные $K_{1}, K_{2}$ (не зависящие от $n, \theta)$ такие, что для оценки максимального правдоподобия $\hat{\theta}_{m}=\hat{\theta}_{m}\left(x^{m}, y^{m}\right)$ выполняется неравенство (см. [4, теорема 5.3])

$$
\sup _{\theta \in[a, b]} \mathbf{P}_{\theta}\left\{\left|\hat{\theta}_{m}-\theta\right|>z\right\} \leqslant K_{1} e^{-K_{2} m z^{2}}, \quad z>0 .
$$

Поэтому для последовательности $\delta(m)=(\ln m) / \sqrt{m}$, начиная с достаточно больших $m$, имеем

$$
\sup _{\theta \in[a, b]} \mathbf{E}_{\theta}\left[\left(\hat{\theta}_{m}-\theta\right)^{2} ; I_{\left|\hat{\theta}_{m}-\theta\right|>\delta(m) / 4}\right] \leqslant \frac{1}{m^{2}} .
$$

Пусть теперь помощник сообщает статистику точные значения первых $m=\sqrt{n}$ величин $\left(y_{1}, \ldots, y_{m}\right)$. С их помощью статистик может построить некоторый интервал длины $\delta(n) / 2$, в котором с большой вероятностью находится параметр $\theta$ (в силу (10) оставшейся малой вероятностью можно пренебречь).

Построим теперь на отрезке $[a, b]$ следующую систему отрезков длины $\delta(n)$. В качестве первого отрезка выберем $[a, a+\delta(n)]$. Далее будем последовательно сдвигать этот отрезок вправо на $\delta(n) / 2$, пока его правый конец не превысит точки $b$. В результате общее число таких отрезков длины $\delta(n)$ в построенной системе не превышает $2(b-a) / \delta(n)+1$. C другой стороны, любой отрезок длины $\delta(n) / 2$ на отрезке $[a, b]$ полностью принадлежит одному из отрезков этой системы.

Далее для каждого отрезка этой системы помощник может использовать свою функцию $f$ и сообщить ее значение статистику. Так как общее число отрезков системы меньше $\sqrt{n}$ (т.е. неэкспоненциально), то это не изменит скорости передачи $R$. Статистик же по первым $\sqrt{n}$ наблюдениям может правильно определить отрезок системы (длины $\delta(n))$, которому принадлежит оцениваемый параметр. После этого он использует соответствующую этому отрезку функцию $f$. Отсюда вытекает справедливость предложения 1.

3 а м е ч а и е. Хотя предложение 1 представляет собой довольно простой и понятный результат, его в свое время не знали авторы работы [2], и это послужило причиной определенных трудностей в [2].

Следствие 1. Справедлива формула

$$
R_{\text {crit }}([a, b])=\sup _{\theta \in[a, b]} R_{\text {crit }}\left(\theta_{\delta(n)}\right)
$$

где $\theta_{\delta(n)}=(\theta-\delta(n), \theta+\delta(n)) \cap[a, b]$. 
В силу предложения 1 и следствия 1 рассматриваемая задача сводится К изучению локального (в малой окрестности истинного значения $\theta$ ) поведения хороших оценок $\tilde{\theta}_{n}$. Ясно, что это поведение будет выражаться в терминах соответствующим образом модифицированной фишеровской информации. Введем необходимые для этого понятия.

Для произвольного разбиения $\left\{\mathscr{Y}_{i} ; i=1, \ldots, N=2^{R n}\right\}$ (определяющего функцию $f_{n}\left(y^{n}\right)$ c $\left.\left|\log f_{n}\right| \leqslant R n\right)$ определим условную и безусловную фишеровскую информацию:

$$
\begin{aligned}
I\left(\theta ; f_{n} \mid x^{n}\right) & =I\left(\theta ;\left\{\mathscr{Y}_{i}\right\} \mid x^{n}\right)=\frac{1}{n} \sum_{i=1}^{N} \frac{p^{\prime 2}\left(\mathscr{Y}_{i} \mid x^{n}, \theta\right)}{p\left(\mathscr{Y}_{i} \mid x^{n}, \theta\right)} \\
I\left(\theta ; f_{n}\right) & =I\left(\theta ;\left\{\mathscr{Y}_{i}\right\}\right)=\sum_{x^{n} \in \mathscr{X}^{n}} \pi\left(x^{n}\right) I\left(\theta ;\left\{\mathscr{Y}_{i}\right\} \mid x^{n}\right) \\
& =\frac{1}{n} \sum_{x^{n} \in \mathscr{X}^{n}} \pi\left(x^{n}\right) \sum_{i=1}^{N} \frac{p^{2}\left(\mathscr{Y}_{i} \mid x^{n}, \theta\right)}{p\left(\mathscr{Y}_{i} \mid x^{n}, \theta\right)}
\end{aligned}
$$

Введем также максимальную достижимую фишеровскую информацию при заданной скорости $R$

$$
I(\theta ; R)=\lim _{n \rightarrow \infty} \sup _{\left|\log f_{n}\right| \leqslant R n} I\left(\theta ; f_{n}\right), \quad R>0 .
$$

Так как в соотношении (8) мы имеем дело только с состоятельными оценками в «регулярном эксперименте» [4], [5], то для любого $R>0$ справедлива формула [2]

$$
\lim _{n \rightarrow \infty} \inf _{\tilde{\theta}_{n}} \sup _{\theta \in c_{\delta(n)}}\left\{n \mathbf{E}_{\theta}\left[\hat{\theta}_{n}\left(x^{n}, f\left(y^{n}\right)\right)-\theta\right]^{2}-\frac{1}{I(\theta ; R)}\right\}=0 .
$$

Ясно также, что для величин $I(\theta ; R)$ и $I(\theta)$ выполняется неравенство

$$
I(\theta ; R) \leqslant I(\theta), \quad R>0 .
$$

Для его доказательства заметим, что для любых $\mathscr{Y}_{i} \subseteq \mathscr{Y}^{n}$ и $x^{n} \in \mathscr{X}^{n}$ в силу неравенства Коши-Буняковского имеем

$$
\begin{aligned}
p^{\prime 2}\left(\mathscr{Y}_{i} \mid x^{n}, \theta\right) & =\left[\sum_{y^{n} \in \mathscr{Y}_{i}} \sqrt{p\left(y^{n} \mid x^{n}, \theta\right)} \frac{p^{\prime}\left(y^{n} \mid x^{n}, \theta\right)}{\sqrt{p\left(y^{n} \mid x^{n}, \theta\right)}}\right]^{2} \\
& \leqslant p\left(\mathscr{Y}_{i} \mid x^{n}, \theta\right) \sum_{y^{n} \in \mathscr{Y}_{i}} \frac{p^{2}\left(y^{n} \mid x^{n}, \theta\right)}{p\left(y^{n} \mid x^{n}, \theta\right)} .
\end{aligned}
$$

Поэтому для любого разбиения $\left\{\mathscr{Y}_{i}\right\}$ и любого $x^{n} \in \mathscr{X}^{n}$ получаем

$$
I\left(\theta ;\left\{\mathscr{Y}_{i}\right\} \mid x^{n}\right)=\frac{1}{n} \sum_{i} \frac{p^{\prime 2}\left(\mathscr{Y}_{i} \mid x^{n}, \theta\right)}{p\left(\mathscr{Y}_{i} \mid x^{n}, \theta\right)} \leqslant \frac{1}{n} \sum_{i} \sum_{y^{n} \in \mathscr{Y}_{i}} \frac{p^{2}\left(y^{n} \mid x^{n}, \theta\right)}{p\left(y^{n} \mid x^{n}, \theta\right)}
$$

что вместе с (12)-(14) дает неравенство (16). 
Поэтому мы приходим к чисто аналитической задаче: при каких $R$ в (16) выполняется равенство? Ясно, что существует определенная критическая скорость $R_{\text {crit }}(\theta) \geqslant 0$ такая, что равенство в (16) выполняется для $R>R_{\text {crit }}(\theta)$ и не выполняется для $R<R_{\text {crit }}(\theta)$. Формально определим $R_{\text {crit }}(\theta) \geqslant 0$ как

$$
R_{\text {crit }}(\theta)=\inf \{R: I(\theta ; R)=I(\theta)\}, \quad \theta \in[a, b] .
$$

2. Оценка сверху для $R_{\text {crit }}(\theta)$ (фишеровская информация для случайно выбранных точек). Чтобы оценить величину $R_{\text {crit }}(\theta)$ сверху, мы оценим снизу величину $I(\theta, R)$ и получим таким образом достаточное условие равенства в соотношении (16).

В свою очередь, чтобы оценить снизу величину $I(\theta ; R)$, мы используем метод «случайного выбора» множеств $\left\{\mathscr{Y}_{i}\right\}$, который уже применялся в первой части работы [1].

Далее мы рассматриваем ДСК из примера 2 в [1] с неизвестной переходной вероятностью $0<\theta \leqslant \frac{1}{2}$ и $\mathscr{X}=\mathscr{Y}=E=\{0 ; 1\}$. Вероятность получить на выходе блок $y^{n}=\left(y_{1}, \ldots, y_{n}\right)$ при условии, что на входе был блок $x^{n}=\left(x_{1}, \ldots, x_{n}\right)$, дается формулой

$$
\mathbf{P}\left(y^{n} \mid x^{n}\right)=(1-\theta)^{n-d\left(x^{n}, y^{n}\right)} \theta^{d\left(x^{n}, y^{n}\right),}
$$

где $d\left(x^{n}, y^{n}\right)$ есть расстояние Хемминга между блоками $x^{n}$ и $y^{n}$. Будем предполагать, что входной блок $x^{n}$ имеет равновероятное априорное распределение на $E^{n}$.

Выберем $N$ натуральных чисел $Y_{1}, \ldots, Y_{N}$ с $Y_{1}+\cdots+Y_{N}=2^{n}$. Каждое $Y_{i}$ есть мощность соответствующей части разбиения $\mathscr{Y}_{i}$. Рассмотрим теперь всевозможные разбиения множества $\mathscr{Y}^{n}$ на $N$ множеств $\left(\mathscr{Y}_{1}, \ldots, \mathscr{Y}_{N}\right)$ с $\left|\mathscr{Y}_{i}\right|=Y_{i}, i=1, \ldots, N$. Другими словами, выберем сначала случайным образом $Y_{1}$ элементов из множества $\mathscr{Y}^{n}$ (это будет множество $\left.\mathscr{Y}_{1}\right)$. Затем из оставшейся части множества $\mathscr{Y}^{n}$ выберем случайным образом следующие $Y_{2}$ элементов (это будет множество $\mathscr{Y}_{2}$ ) и т.д.

В силу симметрии фишеровская информация $I\left(\theta,\left\{\mathscr{Y}_{i}\right\} \mid x^{n}\right)$, усредненная по всем разбиениям $\left\{\mathscr{Y}_{i}\right\}$, не зависит от входного блока $x^{n}$. Поэтому можно считать, что $x^{n}$ есть нулевой блок $0 \in E^{n}$. В результате для усредненной величины $\mathbf{E} I\left(\theta,\left\{\mathscr{Y}_{i}\right\} \mid x^{n}\right)$ имеем из (13)

$$
\mathbf{E} I\left(\theta,\left\{\mathscr{Y}_{i}\right\} \mid x^{n}\right)=\frac{1}{n} \sum_{i=1}^{N} \mathbf{E} \frac{p^{2}\left(\mathscr{Y}_{i} \mid \mathbf{0}, \theta\right)}{p\left(\mathscr{Y}_{i} \mid \mathbf{0}, \theta\right)}=\sum_{i=1}^{N} i\left(\theta, Y_{i}\right), \quad \sum_{i=1}^{N} Y_{i}=2^{n},
$$

где

$$
i(\theta, A)=\frac{1}{n} \mathbf{E} \frac{p^{2}(\mathscr{A} \mid \mathbf{0}, \theta)}{p(\mathscr{A} \mid \mathbf{0}, \theta)}
$$

а последнее усреднение проводится по всем возможным равновероятным множествам $\mathscr{A}$ мощности $A$. 
Исследуем теперь величину $i(\theta, A)$ из (19) и покажем, что она является почти линейной функцией по $A$ при не слишком больших $A$. Для этого рассмотрим сначала случай $0<\theta<\frac{1}{2}$. Выберем некоторое $\theta<r<\frac{1}{2}$ и введем следующие классы $\left\{\mathscr{B}_{i}\right\}$ и $\left\{\mathscr{C}_{i}\right\}$ множеств $\mathscr{A}=\left\{\mathbf{y}_{1}, \ldots, \mathbf{y}_{A}\right\}$, где $\mathbf{y}_{i} \in E^{n}, i=1, \ldots, A$ :

$$
\begin{aligned}
\mathscr{B}_{i} & =\left\{\mathscr{A}: w\left(\mathbf{y}_{i}\right) \leqslant r n\right\}, \quad i=1, \ldots, A, \\
\mathscr{C}_{i} & =\left\{\mathscr{A}: \min _{j \neq i} w\left(\mathbf{y}_{j}\right)>r n\right\}, \quad i=1, \ldots, A,
\end{aligned}
$$

где $w(\mathbf{y})-$ вес (т.е. число единиц) вектора $\mathbf{y}$.

Так как множества $\left\{\mathscr{B}_{i} \cap \mathscr{C}_{i} ; i=1, \ldots, A\right\}$ не пересекаются между собой, то в силу симметрии имеем $\left(\mathbf{E}[\xi ; \mathscr{A}]=\int_{\mathscr{A}} x d F_{\xi}(x)\right)$

$$
\mathbf{E} \frac{p^{\prime 2}(\mathscr{A} \mid \mathbf{0}, \theta)}{p(\mathscr{A} \mid \mathbf{0}, \theta)} \geqslant A \mathbf{E}\left[\frac{p^{\prime 2}(\mathscr{A} \mid \mathbf{0}, \theta)}{p(\mathscr{A} \mid \mathbf{0}, \theta)} ; \mathscr{B}_{1} \cap \mathscr{C}_{1}\right] .
$$

Далее, имеем $\left(M=2^{n}, V_{r n}\right.$ - мощность шара радиуса $r n$ в $\left.E^{n}\right)$

$$
\begin{gathered}
\mathbf{P}\left(\mathscr{B}_{1}\right)=V_{r n} 2^{-n} \\
\mathbf{P}\left(\mathscr{C}_{1} \mid \mathscr{B}_{1}\right)=\left(\begin{array}{c}
M-V_{r n} \\
A-1
\end{array}\right) /\left(\begin{array}{c}
M-1 \\
A-1
\end{array}\right)=\prod_{i=1}^{V_{r n}-1}\left[1-\frac{A-1}{M-V_{r n}-i}\right] \\
\geqslant\left[1-\frac{A-1}{M-2 V_{r n}+1}\right]^{V_{r n}-1} \geqslant 1-\frac{A V_{r n}}{2^{n}-2 V_{r n}} .
\end{gathered}
$$

Заметим, что

$$
\begin{aligned}
\frac{p^{\prime}\left(y^{n} \mid x^{n}, \theta\right)}{p\left(y^{n} \mid x^{n}, \theta\right)} & =\frac{d\left(x^{n}, y^{n}\right)-\theta n}{\theta(1-\theta)}, \quad \frac{p^{\prime}(\mathbf{y} \mid \mathbf{0}, \theta)}{p(\mathbf{y} \mid \mathbf{0}, \theta)}=\frac{w(\mathbf{y})-\theta n}{\theta(1-\theta)} \\
i(\theta, 1) & =\frac{1}{n} \mathbf{E} \frac{p^{\prime 2}(\mathbf{y} \mid \mathbf{0}, \theta)}{p(\mathbf{y} \mid \mathbf{0}, \theta)}=\frac{1}{n} \mathbf{E} \frac{(w(\mathbf{y})-\theta n)^{2}}{\theta^{2}(1-\theta)^{2}} p(\mathbf{y} \mid \mathbf{0}, \theta) \\
& =\frac{2^{-n}}{\theta(1-\theta)}
\end{aligned}
$$

Поэтому для $\left\{\mathbf{y}_{1}, \mathbf{y}_{2}, \ldots, \mathbf{y}_{A}\right\} \in \mathscr{C}_{1}$ справедлива оценка

$$
0 \leqslant \sum_{i=2}^{A} p^{\prime}\left(\mathbf{y}_{i} \mid \mathbf{0}, \theta\right) \leqslant \frac{n}{\theta} \sum_{i=2}^{A} p\left(\mathbf{y}_{i} \mid \mathbf{0}, \theta\right) .
$$

Кроме того, с помощью неравенства Чебышева имеем

$$
\mathbf{P}\left\{\sum_{i=2}^{A} p\left(\mathbf{y}_{i} \mid \mathbf{0}, \theta\right)>z\right\} \leqslant(A-1) 2^{-n} z^{-1}, \quad z>0 .
$$

Введем множество элементарных событий $\mathscr{D}_{z} \subseteq \mathscr{C}_{1}, z>0$ :

$$
\mathscr{D}_{z}=\left\{\left\{\mathbf{y}_{1}, \mathbf{y}_{2}, \ldots, \mathbf{y}_{A}\right\} \in \mathscr{C}_{1}: \sum_{i=2}^{A} p\left(\mathbf{y}_{i} \mid \mathbf{0}, \theta\right) \leqslant z\right\} .
$$


Теперь для $\mathscr{A}=\left\{\mathbf{y}_{1}, \mathbf{y}_{2}, \ldots, \mathbf{y}_{A}\right\} \in \mathscr{D}_{z}$ с помощью (22) имеем

$$
\begin{aligned}
\frac{p^{2}(\mathscr{A} \mid \mathbf{0}, \theta)}{p(\mathscr{A} \mid \mathbf{0}, \theta)} & \geqslant \frac{p^{2}\left(\mathbf{y}_{1} \mid \mathbf{0}, \theta\right)-2 n z \theta^{-1}\left|p^{\prime}\left(\mathbf{y}_{1} \mid \mathbf{0}, \theta\right)\right|}{p\left(\mathbf{y}_{1} \mid \mathbf{0}, \theta\right)+z} \\
& \geqslant \frac{p^{2}\left(\mathbf{y}_{1} \mid \mathbf{0}, \theta\right)}{p\left(\mathbf{y}_{1} \mid \mathbf{0}, \theta\right)}-\frac{z p^{\prime 2}\left(\mathbf{y}_{1} \mid \mathbf{0}, \theta\right)}{p^{2}\left(\mathbf{y}_{1} \mid \mathbf{0}, \theta\right)}-\frac{2 n z\left|p^{\prime}\left(\mathbf{y}_{1} \mid \mathbf{0}, \theta\right)\right|}{\theta p\left(\mathbf{y}_{1} \mid \mathbf{0}, \theta\right)}
\end{aligned}
$$

Поэтому для правой части (20) получаем

$$
\begin{aligned}
\mathbf{E}[ & \left.\frac{p^{\prime 2}(\mathscr{A} \mid \mathbf{0}, \theta)}{p(\mathscr{A} \mid \mathbf{0}, \theta)} ; \mathscr{B}_{1} \cap \mathscr{C}_{1}\right] \geqslant \mathbf{E}\left[\frac{p^{\prime 2}(\mathscr{A} \mid \mathbf{0}, \theta)}{p(\mathscr{A} \mid \mathbf{0}, \theta)} ; \mathscr{B}_{1} \cap \mathscr{D}_{z}\right] \\
\geqslant \mathbf{E} & {\left[\frac{p^{\prime 2}\left(\mathbf{y}_{1} \mid \mathbf{0}, \theta\right)}{p\left(\mathbf{y}_{1} \mid \mathbf{0}, \theta\right)}-\frac{z p^{\prime 2}\left(\mathbf{y}_{1} \mid \mathbf{0}, \theta\right)}{p^{2}\left(\mathbf{y}_{1} \mid \mathbf{0}, \theta\right)}-\frac{2 n z\left|p^{\prime}\left(\mathbf{y}_{1} \mid \mathbf{0}, \theta\right)\right|}{\theta p\left(\mathbf{y}_{1} \mid \mathbf{0}, \theta\right)} ; \mathscr{B}_{1} \cap \mathscr{D}_{z}\right] } \\
=\mathbf{E} & {\left[\frac{p^{\prime 2}\left(\mathbf{y}_{1} \mid \mathbf{0}, \theta\right)}{p\left(\mathbf{y}_{1} \mid \mathbf{0}, \theta\right)}-\frac{z p^{\prime 2}\left(\mathbf{y}_{1} \mid \mathbf{0}, \theta\right)}{p^{2}\left(\mathbf{y}_{1} \mid \mathbf{0}, \theta\right)}\right.} \\
& \left.-\frac{2 n z\left|p^{\prime}\left(\mathbf{y}_{1} \mid \mathbf{0}, \theta\right)\right|}{\theta p\left(\mathbf{y}_{1} \mid \mathbf{0}, \theta\right)} ; \mathscr{B}_{1}\right] \mathbf{P}\left(\mathscr{D}_{z} \mid \mathscr{B}_{1}\right) .
\end{aligned}
$$

Оценим теперь первые три члена из правой части (24) :

$$
\begin{aligned}
\mathbf{E}\left[\frac{p^{\prime 2}\left(\mathbf{y}_{1} \mid \mathbf{0}, \theta\right)}{p\left(\mathbf{y}_{1} \mid \mathbf{0}, \theta\right)} ; \mathscr{B}_{1}\right] & \geqslant \frac{n 2^{-n}}{\theta(1-\theta)}-\frac{n^{2}}{\theta^{2}} \mathbf{E}\left[p\left(\mathbf{y}_{1} \mid \mathbf{0}, \theta\right) ; \mathscr{B}_{1}^{c}\right] \\
& =\frac{n 2^{-n}}{\theta(1-\theta)}-\frac{n^{2} 2^{-n}}{\theta^{2}} \mathbf{P}_{\theta}\left(\mathscr{B}_{1}^{c}\right), \\
\mathbf{E}\left[\frac{p^{\prime 2}\left(\mathbf{y}_{1} \mid \mathbf{0}, \theta\right)}{p^{2}\left(\mathbf{y}_{1} \mid \mathbf{0}, \theta\right)} ; \mathscr{B}_{1}\right] & \leqslant \frac{n^{2} V_{r n} 2^{-n}}{\theta^{2}}, \\
\mathbf{E}\left[\frac{\left|p^{\prime}\left(\mathbf{y}_{1} \mid \mathbf{0}, \theta\right)\right|}{p\left(\mathbf{y}_{1} \mid \mathbf{0}, \theta\right)} ; \mathscr{B}_{1}\right] & \leqslant \frac{n V_{r n} 2^{-n}}{\theta} .
\end{aligned}
$$

Далее, с помошью (21) и (23) для $r \leqslant \frac{1}{2}$ и $A \geqslant 2$ имеем

$$
\begin{aligned}
\mathbf{P}\left(\mathscr{D}_{z} \mid \mathscr{B}_{1}\right) & \geqslant \mathbf{P}\left(\mathscr{C}_{1} \mid \mathscr{B}_{1}\right)-\mathbf{P}\left\{\sum_{i=2}^{A} p\left(\mathbf{y}_{i} \mid \mathbf{0}, \theta\right)>z\right\} \\
& \geqslant 1-A 2^{-n}\left(2 V_{r n}+z^{-1}\right) .
\end{aligned}
$$

Поэтому из (20) и (24) получаем

$$
\mathbf{E} \frac{p^{\prime 2}(\mathscr{A} \mid \mathbf{0}, \theta)}{p(\mathscr{A} \mid \mathbf{0}, \theta)} \geqslant \frac{A n 2^{-n}}{\theta(1-\theta)}\left[1-\frac{n \mathbf{P}_{\theta}\left(\mathscr{B}_{1}^{c}\right)+3 n z V_{r n}}{\theta}-\frac{A\left(2 V_{r n}+z^{-1}\right)}{2^{n}}\right] .
$$

Нам осталось оценить величины $\mathbf{P}_{\theta}\left(\mathscr{B}_{1}^{c}\right)$ и $V_{r n}$ в правой части $(25)$ и выбрать параметры $r, z$. Сначала, используя стандартные оценки для 
биномиальных коэффициентов [6], [7] и формулу Тейлора для функции $h(r)[1$, п. 2.2], имеем

$$
\begin{aligned}
\mathbf{P}_{\theta}\left(\mathscr{B}_{1}^{c}\right) & =\sum_{i>r n}\left(\begin{array}{c}
n \\
i
\end{array}\right) \theta^{i}(1-\theta)^{n-i} \leqslant \frac{\theta^{r n+1}(1-\theta)^{n(1-r)} 2^{n h(r)}}{2(r-\theta) \sqrt{n r}} \\
& \leqslant \frac{\theta}{2(r-\theta) \sqrt{n r}} \exp \left\{-2 n(r-\theta)^{2}\right\}, \quad \theta<r \leqslant \frac{1}{2} \\
V_{r n} & \leqslant \frac{\sqrt{1-r}}{2(1-2 r) \sqrt{r n}} 2^{n h(r)}, \quad r<\frac{1}{2} .
\end{aligned}
$$

Полагая теперь $r=\theta+\sqrt{(\ln n) / n}, z=1 /\left(n^{2} V_{r n}\right)$, получаем

$$
\begin{aligned}
\mathbf{P}_{\theta}\left(\mathscr{B}_{1}^{c}\right) & \leqslant n^{-2} \sqrt{\frac{\theta}{\ln n}}, \quad \theta+\sqrt{\frac{\ln n}{n}}<\frac{1}{2}, \\
V_{r n} & \leqslant \frac{2^{n h(\theta)}}{2 n^{2}(1-2 \theta-2 \sqrt{(\ln n) / n}) \sqrt{n \theta}}\left(\frac{1-\theta}{\theta}\right)^{\sqrt{n \ln n}} .
\end{aligned}
$$

Из (25) и (26) вытекает следующий результат, представляющий также самостоятельный интерес.

Предложение 2. Для величины $i(\theta, A), 0<\theta<\frac{1}{2}$, справедливы оченки

$$
\begin{aligned}
1- & \frac{1}{n \sqrt{\theta \ln n}}-\frac{3}{n \theta}-\frac{A 2^{n h(\theta)-n}}{(1-2 \theta-2 \sqrt{(\ln n) / n}) \sqrt{\theta n}}\left(\frac{1-\theta}{\theta}\right)^{\sqrt{n \ln n}} \\
& \leqslant \frac{\theta(1-\theta) 2^{n}}{A} i(\theta, A) \leqslant 1, \quad \theta+\sqrt{\frac{\ln n}{n}}<\frac{1}{2} .
\end{aligned}
$$

Вернемся теперь к соотношению (18). Пусть мощность $Y_{i}$ каждой части разбиения $\mathscr{Y}_{i}$ удовлетворяет условию

$$
Y_{i} \leqslant 2^{n-n h(\theta)}\left(\frac{\theta}{1-\theta}\right)^{\sqrt{n \ln n}}, \quad i=1, \ldots, N .
$$

Для таких разбиений $\left\{\mathscr{Y}_{i}\right\}$ в силу (27) равномерно по $\theta \in[a, b], 0<a<$ $b<\frac{1}{2}$, имеем

$$
\mathbf{E} I\left(\theta ;\left\{\mathscr{Y}_{i}\right\}\right)=\frac{n}{\theta(1-\theta)}(1+o(1)), \quad n \rightarrow \infty .
$$

Ясно, что для существования набора $\left\{Y_{i}, i=1, \ldots, N\right\}$, удовлетворяющего условию (28), достаточно, чтобы для общего числа $N$ частей разбиения было выполнено условие

$$
N \geqslant 2^{n h(\theta)}\left(\frac{1-\theta}{\theta}\right)^{\sqrt{n \ln n}}
$$

Заметим также, что для величины $I(\theta ; R)$ выполняется свойство

$$
I(\theta ; R)=I(1-\theta ; R), \quad 0<\theta<1, R>0 .
$$


В результате из (11) и (27)-(30) получаем

Следствие 2. Справедливо соотношение

$$
I(\theta ; R)=I(\theta) \quad \partial \Omega s R \geqslant h(\theta), 0<\theta<1 ;
$$

и, следовательно,

$$
R_{\text {crit }}(\theta) \leqslant h(\theta), \quad R_{\text {crit }}([a, b]) \leqslant \max _{x \in[a, b]} h(x) .
$$

3. Точка $\theta=\frac{1}{2}$. В случае точки $\theta=\frac{1}{2}$ вычисления, связанные с фишеровской информацией, значительно упрощаются, что позволяет найти точный вид функции $I\left(\theta=\frac{1}{2} ; R\right)$ для всех $R>0$.

Сначала из определения (13) имеем

$$
I\left(\theta ;\left\{\mathscr{Y}_{i}\right\}\right)=n^{-1} 2^{-n} \sum_{x^{n} \in E^{n}} \sum_{i=1}^{N} \frac{p^{\prime 2}\left(\mathscr{Y}_{i} \mid x^{n}, \theta\right)}{p\left(\mathscr{Y}_{i} \mid x^{n}, \theta\right)}=\sum_{i=1}^{N} i\left(\theta, \mathscr{Y}_{i}\right),
$$

где обозначено

$$
\begin{aligned}
i(\theta, \mathscr{A}) & =\frac{2^{-n}}{n} \sum_{x^{n} \in E^{n}} \frac{p^{\prime 2}\left(\mathscr{A} \mid x^{n}, \theta\right)}{p\left(\mathscr{A} \mid x^{n}, \theta\right)} \\
& =\frac{2^{-n}}{n \theta^{2}(1-\theta)^{2}} \sum_{x^{n} \in E^{n}} \frac{\left[\sum_{y^{n} \in \mathscr{A}}\left(d\left(x^{n}, y^{n}\right)-n \theta\right) p\left(y^{n} \mid x^{n}, \theta\right)\right]^{2}}{\sum_{y^{n} \in \mathscr{A}} p\left(y^{n} \mid x^{n}, \theta\right)} .
\end{aligned}
$$

При $\theta=\frac{1}{2}$ величина $i(\theta, \mathscr{A})$ из (34) принимает вид $(A=|\mathscr{A}|)$

$$
\begin{aligned}
i\left(\frac{1}{2}, \mathscr{A}\right) & =\frac{2^{4-2 n}}{n A} \sum_{y_{1}^{n} \in \mathscr{A}} \sum_{y_{2}^{n} \in \mathscr{A}} \sum_{x^{n} \in E^{n}}\left(d\left(y_{1}^{n}, x^{n}\right)-\frac{n}{2}\right)\left(d\left(y_{2}^{n}, x^{n}\right)-\frac{n}{2}\right) \\
& =A 2^{2-n}\left\{1-\frac{2}{n A^{2}} \sum_{y_{1}^{n} \in \mathscr{A}} \sum_{y_{2}^{n} \in \mathscr{A}} d\left(y_{1}^{n}, y_{2}^{n}\right)\right\},
\end{aligned}
$$

где использовались вычисления $\left(d=d\left(y_{1}^{n}, y_{2}^{n}\right)\right)$

$$
\begin{aligned}
& \sum_{x^{n} \in E^{n}}\left(d\left(y_{1}^{n}, x^{n}\right)-\frac{n}{2}\right)\left(d\left(y_{2}^{n}, x^{n}\right)-\frac{n}{2}\right) \\
& =\sum_{w=0}^{d} \sum_{v=0}^{n-d}\left(\begin{array}{c}
d \\
w
\end{array}\right)\left(\begin{array}{c}
n-d \\
v
\end{array}\right)\left(w+v-\frac{n}{2}\right)\left(d-w+v-\frac{n}{2}\right) \\
& =2^{d-2} \sum_{v=0}^{n-d}\left(\begin{array}{c}
n-d \\
v
\end{array}\right)\left[4 v^{2}+4 v(d-n)+d(d-1)-n(2 d-n)\right] \\
& =2^{n-2}\left(n-2 d\left(y_{1}^{n}, y_{2}^{n}\right)\right) .
\end{aligned}
$$

Для того чтобы найти максимум в правой части (35) при заданной мощности $A$, необходимо найти минимум (по всем множествам $\mathscr{A}$ ) 
выражения $2\left(n A^{2}\right)^{-1} \sum_{y_{1}^{n} \in \mathscr{A}} \sum_{y_{2}^{n} \in \mathscr{A}} d\left(y_{1}^{n}, y_{2}^{n}\right)$. Более того, нас будет интересовать асимптотическое (при $n \rightarrow \infty$ ) поведение этого минимума, когда $A \sim 2^{r n}, 0<r<\frac{1}{2}$. Известно [8], что асимптотически этот минимум достигается, когда множество $\mathscr{A}$ является шаром $B_{\alpha n} \subset E^{n}$ некоторого радиуса $\alpha n, 0<r<\frac{1}{2}$. Так как $A \sim 2^{r n} \sim\left|B_{\alpha n}\right| \sim 2^{n h(\alpha)}$, $h(x)=-x \log x-(1-x) \log (1-x)$, то

$$
h(\alpha)=r=\frac{\log A}{n} .
$$

Для того чтобы вычислить сумму в правой части (35), представим себе, что все блоки $y^{n} \in \mathscr{A}$ записаны в виде матрицы размера $A \times n$ (т.е. каждая строка матрицы есть некоторый блок $y^{n}$ ). Пусть, далее, $m_{i}$, $i=1, \ldots, n$, есть число единиц в $i$-м столбце этой матрицы. Тогда имеем

$$
\frac{2}{n A^{2}} \sum_{y_{1}^{n} \in \mathscr{A}} \sum_{y_{2}^{n} \in \mathscr{A}} d\left(y_{1}^{n}, y_{2}^{n}\right)=\frac{4}{n A^{2}} \sum_{i=1}^{n} m_{i}\left(A-m_{i}\right) .
$$

Для общего числа единиц в матрице имеем

$$
\sum_{i=1}^{n} m_{i}=\sum_{j=0}^{\alpha n} j\left(\begin{array}{l}
n \\
j
\end{array}\right)=\alpha n\left|B_{\alpha n}\right|(1+o(1)) .
$$

Поэтому

$$
m_{1}=\cdots=m_{n}=\alpha\left|B_{\alpha n}\right|(1+o(1)), \quad n \rightarrow \infty,
$$

и, следовательно, для шара $\mathscr{A}$ имеем

$$
\frac{2}{n A^{2}} \sum_{y_{1}^{n} \in \mathscr{A}} \sum_{y_{2}^{n} \in \infty} d\left(y_{1}^{n}, y_{2}^{n}\right)=4 \alpha(1-\alpha)(1+o(1)), \quad n \rightarrow \infty .
$$

В результате из (35) получаем

$$
\max _{\mathscr{A}:|\mathscr{A}|=A} i\left(\frac{1}{2}, \mathscr{A}\right)=A 2^{2-n}(1-2 \alpha)^{2}(1+o(1)), \quad n \rightarrow \infty
$$

где $\alpha$ определено в (36).

Для того чтобы с помощью формулы (38) получить необходимый результат для всего пространства $E^{n}$, нам потребуется следующее усиление леммы о «покрытиях» из [1].

Лемма. Пусть $\mathscr{X}=\left\{\mathbf{x}_{1}, \ldots, \mathbf{x}_{X}\right\} \subset E^{n}$ есть произвольное множество мощности $X \leqslant 2^{n-2}$ и $\left.K=\right](n+1) 2^{n} / X$. Тогда существуют $K$ «сдвигов» $\left\{\mathbf{y}_{1}, \ldots, \mathbf{y}_{K}\right\} \subset E^{n}$ таких, что:

1) множества $\mathscr{X}_{i}=\mathscr{X}+\mathbf{y}_{i}, i=1, \ldots, K$, покрывают пространство $E^{n}$, причем любая точка $\mathbf{x} \in E^{n}$ покрыта не более $4 n$ раз;

2) если $X \geqslant 6 n^{2}$, то существует система непересекающихся множеств $\mathscr{Y}_{i} \subseteq \mathscr{X}_{i}, i=1, \ldots, K$, покрывающих пространство $E^{n}, c$ $\left|\mathscr{Y}_{i}\right| \geqslant X /(8 n), i=1, \ldots, K$. 
Д ок а з а т ль с т в о. Пусть $K, m$ - произвольные натуральные числа. Чтобы получить первое утверждение леммы, выберем $K$ сдвигов случайным независимым образом (с возвращениями). Тогда имеем

$$
\begin{aligned}
P_{0}= & \operatorname{Pr}\left\{\begin{array}{c}
\text { найдется непокрытая точка } \mathbf{x} \in E^{n}, \text { либо } \\
\text { найдется точка } \mathbf{x} \in E^{n}, \text { покрытая более чем } m \text { раз }
\end{array}\right\} \\
\leqslant & 2^{n} \operatorname{Pr}\{\text { точка } 0 \text { не покрыта }\} \\
& +2^{n} \operatorname{Pr}\{\text { точка } 0 \text { покрыта более чем } m \text { раз }\}=P_{1}+P_{2} .
\end{aligned}
$$

Для любого $K>(n+1) 2^{n} \ln 2 / X$ получаем

$$
P_{1}=2^{n}\left(1-X 2^{-n}\right)^{K} \leqslant \exp \left\{-X K 2^{-n}+n \ln 2\right\}<\frac{1}{2} .
$$

Далее, используя известные оценки для биномиальных коэффициентов [7], имеем $\left(M=2^{n}\right)$

$$
\begin{aligned}
\ln P_{2} & =\ln \left\{M \sum_{i=m+1}^{K}\left(\begin{array}{c}
K \\
i
\end{array}\right)\left(\frac{X}{M}\right)^{i}\left(1-\frac{X}{M}\right)^{K-i}\right\} \\
& \leqslant \ln M+K h_{e}\left(\frac{m+1}{K}\right)+K \ln \left(1-\frac{X}{M}\right)-(m+1) \ln \frac{M-X}{X} \\
& \leqslant \ln M-\frac{K X}{M}+(m+1) \ln \frac{e K X}{(m+1)(M-X)},
\end{aligned}
$$

где для функции $h_{e}(x)=-x \ln x-(1-x) \ln (1-x)$ использовалась оценка $h_{e}(x) \leqslant x \ln (e / x)$. Теперь для $K$, определенного в условии леммы, и $m=4 n$ получаем $P_{2} \leqslant \frac{1}{2}$. В итоге для таких $K, m$ имеем $P_{0}<1$, и поэтому существует набор сдвигов, удовлетворяющий первому утверждению леммы.

Чтобы доказать второе утверждение леммы, возьмем какую-либо точку $x \in E^{n}$. Она покрыта не более чем $m$ множествами $\mathscr{X}_{i}$. Отнесем ее к одному из этих множеств случайным равновероятным образом. Проделаем эту процедуру для всех точек $x \in E^{n}$. В результате мы получим систему непересекающихся множеств $\mathscr{Y}_{i} \subseteq \mathscr{X}_{i}, i=1, \ldots, K$, покрывающих пространство $E^{n}$. Для нее при $2 m N \leqslant X$ имеем

$$
\begin{aligned}
Q_{0} & =\operatorname{Pr}\left\{\text { найдется множество } \mathscr{Y}_{i} \text { с }\left|\mathscr{Y}_{i}\right| \leqslant N\right\} \\
& \leqslant K \mathbf{P}\left\{\left|\mathscr{Y}_{1}\right| \leqslant N\right\} \leqslant K \sum_{j=0}^{N}\left(\begin{array}{c}
X \\
j
\end{array}\right) m^{-j}\left(1-\frac{1}{m}\right)^{X-j} \\
& \leqslant K\left(1-\frac{1}{m}\right)^{X}(m-1)^{-N} \exp \left\{X h_{e}\left(\frac{N}{X}\right)\right\} \\
& <K \exp \left\{N \ln \frac{e X}{(m-1) N}-\frac{X}{m}\right\} \leqslant K e^{-X /(2 m)}<1,
\end{aligned}
$$


если $X \geqslant 6 n^{2}$. Следовательно, существует набор сдвигов, удовлетворяющий второму утверждению леммы.

Вернемся теперь $\mathrm{x}$ соотношению (33), и пусть $\mathscr{A}=B_{\alpha n}(\mathbf{0})$ с $|\mathscr{A}|=$ $A \simeq 2^{r n}$. В силу леммы существуют $N=(n+1) 2^{n} / A \simeq n 2^{n(1-r)}$ сдвигов $\left\{\mathbf{y}_{1}, \ldots, \mathbf{y}_{N}\right\} \subset E^{n}$ таких, что шары $B_{\alpha n}\left(\mathbf{y}_{i}\right)=\mathscr{A}+\mathbf{y}_{i}, i=1, \ldots, N$, покрывают все пространство $E^{n}$. В силу второго утверждения леммы существуют непересекающиеся множества $\mathscr{Y}_{i} \subset B_{\alpha n}\left(\mathbf{y}_{i}\right), i=1, \ldots, N$, которые по-прежнему покрывают все пространство $E^{n}$, причем каждое множество $\mathscr{Y}_{i}$ имеет мощность $\left|\mathscr{Y}_{i}\right| \geqslant\left|B_{\alpha n}\right| /(8 n)$.

Нам осталось показать, что соотношение (37) остается верным и для таких множеств $\left\{\mathscr{Y}_{i}\right\}$. Для этого заметим, что в шаре $B_{\alpha n}$ число упорядоченных пар $\left(y_{1}^{n}, y_{2}^{n}\right)$, для которьх $\left|d\left(y_{1}^{n}, y_{2}^{n}\right)-2 \alpha(1-\alpha) n\right|>\sqrt{n \ln n}$, не превышает $\left|B_{\alpha n}\right|^{2} n^{-3}$. Так как $\mathscr{Y}_{i} \subset B_{\alpha n}$ и общее число упорядоченных пар $\left(y_{1}^{n}, y_{2}^{n}\right)$ в $\mathscr{Y}_{i}$ имеет порядок $\left|B_{\alpha n}\right|^{2} n^{-2}$, то для «основной массы» этих пар выполняется соотношение $\left|d\left(y_{1}^{n}, y_{2}^{n}\right)-2 \alpha(1-\alpha) n\right| \leqslant \sqrt{n \ln n}$, откуда получаем

$$
\begin{aligned}
& \frac{2}{n Y_{i}^{2}} \sum_{y_{1}^{n} \in \mathscr{Y}_{i}} \sum_{y_{2}^{n} \in \mathscr{Y}_{i}} d\left(y_{1}^{n}, y_{2}^{n}\right)=4 \alpha(1-\alpha)(1+o(1)), \quad i=1, \ldots, N, \\
& i\left(\frac{1}{2}, \mathscr{Y}_{i}\right)=Y_{i} 2^{2-n}(1-2 \alpha)^{2}(1+o(1)), \quad i=1, \ldots, N, n \rightarrow \infty .
\end{aligned}
$$

Так как $\sum_{i} Y_{i}=2^{n}$, то из (33) и (40) следует

$$
I\left(\frac{1}{2} ;\left\{\mathscr{Y}_{i}\right\}\right)=4(1-2 \alpha)^{2} .
$$

В итоге из (33)-(41) получаем

Предложение 3. Функиия $I\left(\frac{1}{2} ; R\right)$ определяется соотношением

$$
I\left(\frac{1}{2} ; R\right)=4(1-2 \alpha)^{2}, \quad 0<R<1,
$$

где $h(\alpha)=1-R$. В частности, $R_{\text {crit }}\left(\frac{1}{2}\right)=0$.

Для сравнения найдем также величину $i\left(\frac{1}{2}, A\right)$, представляющую собой усреднение величины $i\left(\frac{1}{2}, \mathscr{A}\right)$ по всем случайно выбранным множествам $\mathscr{A}$ мощности $A$. При этом можно считать, что множество $\mathscr{A}$ имеет вид $\mathscr{A}=\left\{y_{1}^{n}=\mathbf{0}, y_{2}^{n}, \ldots, y_{A}^{n}\right\}$. Тогда имеем

$$
A^{-2} \mathbf{E} \sum_{y_{1}^{n} \in \mathscr{A}} \sum_{y_{2}^{n} \in \mathscr{A}} d\left(y_{1}^{n}, y_{2}^{n}\right)=A^{-1} \mathbf{E} \sum_{y \in \mathscr{A}} w(y)=\frac{n}{2}\left(1-\frac{1}{A}\right)\left(1+\frac{1}{2^{n}-1}\right) \text {. }
$$

Поэтому из (35) получаем

$$
i\left(\frac{1}{2}, A\right)=\mathbf{E} i\left(\frac{1}{2}, \mathscr{A}\right)=2^{2-n}\left(1-\frac{A-1}{2^{n}-1}\right) .
$$


В результате из (33) для $0 \leqslant R<1$ имеем

$$
\mathbf{E} I\left(\frac{1}{2} ;\left\{\mathscr{A}_{i}\right\}\right)=2^{2-n}(N-1)=2^{2-(1-R) n}\left(1-2^{-R n}\right) \rightarrow 0, \quad n \rightarrow \infty .
$$

Это означает, что при $\theta=\frac{1}{2}$ случайный выбор множеств $\left\{\mathscr{A}_{i}\right\}$ является очень плохим (получаемая фишеровская информация даже стремится к 0 при $n \rightarrow \infty$ ). Можно высказать предположение, что случайный выбор множеств $\left\{\mathscr{A}_{i}\right\}$ дает асимптотически оптимальные результаты только при $R>R_{\text {crit }}(\theta)$. В случае $R<R_{\text {crit }}(\theta)$ оптимальное множество $\mathscr{A}_{i}$, видимо, представляет собой набор «хорошо разделенных» шаров определенного радиуса. В этом состоит некоторое отличие ситуации в этой задаче от традиционной ситуации в теории информации (где «средний» код всегда является достаточно хорошим).

\section{СПИСОК ЛИТЕРАТУРЫ}

1. Бурнашев М. В., Амари ШШ., Хая Т. С. О некоторых задачах проверки гипотез с информационными ограничениями. - Теория вероятн. и ее примен., 2000, т. 45, B. 4 , c. $625-638$.

2. Ahlswede R., Burnashev M.V. On minimax estimation in the presence of side information about remote data. - Ann. Statist., 1990, v. 18, № 1, p. 141-171.

3. Han T.S., Amari S. Parameter estimation with multiterminal data compression. IEEE Trans. Inform. Theory, 1995, v. 41, № 6, p. 1802-1833.

4. Ибрагимов И. А., Хасьмикский Р. З. Асимптотическая теория оценивания. М.: Наука, 1979, 528 c.

5. Le Cam L. Asymptotic Methods in Statistical Decision Theory. New York: SpringerVerlag, $1986,742 \mathrm{p}$.

6. Фаяо $P$. Передача информации. Статистическая теория связи. М.: Мир, 1965, 439 c.

7. Галлагер $P$. Теория информации и надежная связь. М.: Советское радио, 1974, $720 \mathrm{c}$.

8. Ahlswede R., Althofer I. The asymptotic behavior of diameters in the average. J. Combin. Theory, Ser. B, 1994, v. 61, № 2, p. 167-177. 